\title{
The Concurrency Workbench with Priorities
}

\author{
Claus Torp Jensen ctjmrr@daimi.dk \\ Computer Science Department \\ Aarhus University \\ DK 8000 Aarhus C.
}

\begin{abstract}
This paper presents an extension of Milner's $C C S$ with a priority choice operator accompanied by a suitable notion of bisimulation and a characterizing modal logic. It is implemented in an extension of the analysis tool the Edinburgh-Sussex Concurrency Workbench.
\end{abstract}

\section{Background}

Many everyday systems include alternative activities with different priorities. Consider a nuclear reactor - if something goes wrong, the shutdown of the reactor should have the highest priority of all. Some recent programming languages (like OCCAM) include the possibility of giving some actions priority over others, but when modelling in CCS there is no way in which one could force an agent to choose a particular one of the two subagents in a sum, so what is actually needed in $C C S$, is a new constructor.

A priority choice constructor $>$ named PRISUM (it has some resemblance to the construction PRI ALT in OCCAM - see OCC[84]) is chosen, which gives the left agent preference to the right, and otherwise behaves like a sum. So $A>B$ means: "Perform as $A$ if you can - if no transitions for $A$ are possible, then perform as $B^{\prime \prime}$

This construct is extendable to multiple levels of priorities where the PRISUM construct chooses a transition for the leftmost agent possible. The control system for the nuclear reactor could now be modelled by shutdown.nil $>G O$.

The theoretical part of this paper is based on Milner's CCS and on work done by Juanito Camilleri (Camilleri[90]). Camilleri's work on priorities is reformulated and simplified, so that it now constitutes a practical basis for an implementation of a state space analysis tool. A key improvement of the present formulation is that the number of possible transitions is dramatically decreased without losing information. The forthcoming paper Camilleri/Winskel[91] is closely related, and presents a complete equational proof system for the equivalence used here, although based on a different operational semantics. The actual implementation is done by modifying the existing analysis tool the EdinburghSussex Concurrency Workbench (called CW - see CW[1-3] for details). The changes are conservative, so that without priorities the system behaves exactly as it did before. All proofs are left out because of lack of space, but will appear in a forthcoming report. 


\section{Construction of transition-system for CCS with priorities}

In the rest of the paper, let $A c t$ be the set of actions (including $\tau$ ), $A$ the set of input actions (not necessarily physical input actions), Agt the set of $C C S^{>}$agents, and for a set $S$ let $S^{n}$ be the set of vectors of length $n$ over $S$ and $P(S)$ the powerset of $S$.

\subsection{The extended language}

If $X \in V S$, where $V S$ is a set of variables (restriction to just one variable would prohibit nesting of fixpoints), $C C S^{>}$expressions have the following regular syntax:

$$
\begin{aligned}
& G::=X \mid \text { nil }|a . E| \tau . E|G \backslash L| G[f]|G+G| G>G|(G \mid G)| \text { fix }(X=G) \\
& E::=X \mid \text { nil }|\alpha . E| E \backslash L|E[f]| E+E|G>G|(E \mid E) \mid \text { fix }(X=E)
\end{aligned}
$$

where the symbols from $C C S$ have their usual meaning, $a \in A, L \in P(A)$ and $\alpha \in A c t$. $f$ is an injective relabeling function. Only guarded recursions are allowed.

The syntax restricts guards (the agents in priority constructs) so that they do not contain output actions on the top level (this is consistent with OCCAM). The reason is, that looking at an agent like $A|B \equiv(\alpha . n i l>\bar{\beta} . n i l)|(\beta . n i l>\bar{\alpha} . n i l)$ it is not clear what its transitions should be. $A$ can do an $\alpha$ transition in a handshake with $B$, but this can only be the case if $B$ cannot do a $\beta$ transition, which can only be the case if a possible $\alpha$ transition for $A$ prevents the $\bar{\beta}$ transition. Similarly $B$ can do a $\beta$ transition if $A$ cannot do an $\alpha$ transition, which can only be the case if a possible $\beta$ transition for $B$ prevents the $\bar{\alpha}$ transition. What choice should one make then?

Because all recursions are guarded, one single set of recursion variables is sufficient. In a guard, any recursion variable must be guarded by an input action. Accordingly it is never possible to introduce an output action on the top level by substitution for variables (output actions in guards are allowed if they are guarded by an input action).

\subsection{The environment's influence on behaviour}

What kind of information about the environment is actually relevant, when it comes to deciding the possible transitions for an agent? The answer is information about what the environment cannot do, and this is captured in the notion of refusal sets. A refusal set is defined to be a set of input actions, for which it is known that the environment can't perform any of the matching output actions.

Since all actions in $C C S$ are basically performed in handshakes, one expects for $A, B \in$ Agt and $\alpha \in$ Act: "If $A$ 's environment refuses to perform an $\bar{\alpha}$ transtion, then $A$ can't perform an $\alpha$-transition". Accumulating over the possible actions for $A$, one expects: "If the environment of $A>B$ refuses to match any of $A$ 's transitions, then $B$ can be allowed to perform its transitions".

\subsection{Goals for the transition system}

When automated analysis is the goal, the transition system for $C C S^{>}$should be computationally tractable, and preferably intuitively understandable. 
Given the informal definition of refusal sets above, it is immediately observable that a given transition would be possible under a very large (actually infinite) number of different refusal sets. This would prevent any analysis using all possible transitions for finite state agents (which is commonly used), so some kind of simplification is needed. The solution is to use transitions of the form $\vdash_{R} A \stackrel{\alpha}{\rightarrow} A^{\prime}$ understood as meaning "In an environment which refuses at least $R$ (and possibly more actions), and is prepared to match $\alpha$, the agent $A$ can perform $\alpha$ to become $A^{\prime \prime}$.

Thus, the subscripted refusal set should be the weakest condition for a given derivation. It is not my intention to allow a transition to occur under all imaginable refusal sets - in order to get an efficient implementation, I just want the smallest ones.

In view of the above description, one would expect of a suitable transition system that $\vdash_{R} A \stackrel{\alpha}{\rightarrow} A^{\prime} \Rightarrow \alpha \notin R$.

\subsection{Transition rules}

\subsubsection{Preliminaries}

In order to define the transition system, two functions on agents are needed (note that these functions only work properly on guarded recursions).

First of all, it is necessary to determine the possible actions of a guard (always input actions or taus), in order to express what the environment cannot be allowed to do, if the righthand agent in a PRISUM construct is to perform any actions.

Definition 1 Define inActs on guards by the following structural induction:

$$
\begin{aligned}
& \text { inActs }(n i l)=\emptyset \\
& \text { inActs }(a . A)=\{a\} \\
& \text { inActs }(\tau . A)=\{\tau\} \\
& \text { inActs }\left(A_{1}+A_{2}\right)=\operatorname{inActs}\left(A_{1}\right) \cup \operatorname{inActs}\left(A_{2}\right) \\
& \text { inActs }(A \backslash L)=i n A c t s(A) \backslash L \\
& \text { inActs }(A[f])=(\text { inActs }(A))[f] \\
& \text { inActs }\left(G_{1}>G_{2}\right)=\operatorname{inActs}\left(G_{1}\right) \cup \operatorname{inActs}\left(G_{2}\right) \\
& \text { inActs }\left(A_{1} \mid A_{2}\right)=\text { inActs }\left(A_{1}\right) \cup \text { inActs }\left(A_{2}\right) \\
& \text { inActs }(f i x(X=A))=\operatorname{in} A c t s(A)
\end{aligned}
$$

where $X \in V S$. Observe that $\tau$ can never be in any refusal set, since refusal sets consist of the input actions which the environment cannot match (and there is no such thing as an $\bar{\tau}$ action) - this expresses the fact that the internal $\tau$ action is always possible (and therefore always prevents low priority actions), and is similar to the SKIP option in the PRI ALT construction in OCCAM.

(9) is so simple because all recursions are guarded. Since only the top level actions are interesting, it is not necessary to unfold.

It might be interesting to notice, that the definition of inActs is independent of the environment. This is due to the fact that in $A>B$ although the environment does influence the possible transitions for $A$, it has to refuse all the handshakes, if $B$ is to be allowed to perform any actions.

One also needs the possible output actions for an agent (note that these are always 
independent of the environment, since output actions are not allowed in guards), because in a parallel composition $A \mid B$ the environment for $A$ is the external environment together with $B$.

Definition 2 Define outActs on agents by the following structural induction:

$$
\begin{aligned}
& \text { outActs }(\text { nil })=\emptyset \\
& \text { outActs }(a \cdot A)=\emptyset \\
& \text { outActs }(\bar{a} \cdot A)=\{a\} \\
& \text { outActs }(\tau \cdot A)=\emptyset \\
& \text { outActs }\left(A_{1}+A_{2}\right)=\text { outActs }\left(A_{1}\right) \cup \text { outActs }\left(A_{2}\right) \\
& \text { outActs }(A \backslash L)=\text { outActs }(A) \backslash L \\
& \text { outActs }(A[f])=(\text { outActs }(A))[f] \\
& \text { outActs }\left(G_{1}>G_{2}\right)=\emptyset \\
& \text { outActs }\left(A_{1} \mid A_{2}\right)=\text { outActs }\left(A_{1}\right) \cup \text { outActs }\left(A_{2}\right) \\
& \text { outActs }(f i x(X=A))=\operatorname{out} A c t s(A)
\end{aligned}
$$

Note regarding (17), that output-actions are not allowed in guards.

\subsubsection{The rules}

The transition rules are now defined as follows:

$$
\begin{gathered}
\vdash_{\theta} \alpha . A \stackrel{\alpha}{\rightarrow} A \\
\frac{\vdash_{R} A \stackrel{\alpha}{\rightarrow} A^{\prime}}{\vdash_{R} A+B \stackrel{\alpha}{\rightarrow} A^{\prime}} \\
\frac{\vdash_{R} B \stackrel{\alpha}{\rightarrow} B^{\prime}}{\vdash_{R} A+B \stackrel{\alpha}{\rightarrow} B^{\prime}} \\
\frac{\vdash_{R} A \stackrel{\alpha}{\rightarrow} A^{\prime}(\alpha \notin L \cup \bar{L})}{\vdash_{R \backslash L} A \backslash L \stackrel{\alpha}{\rightarrow} A^{\prime} \backslash L} \\
\frac{\vdash_{R} A \stackrel{\alpha}{\rightarrow} A^{\prime}}{\vdash_{R[f]} A[f] \stackrel{f(\alpha)}{\rightarrow} A^{\prime}[f]} \\
\frac{\vdash_{R} G_{1} \stackrel{a}{\rightarrow} G_{1}^{\prime}}{\vdash_{R} G_{1}>G_{2} \stackrel{\rightarrow}{\rightarrow} G_{1}^{\prime}} \\
\frac{\vdash_{R} G_{2} \stackrel{\alpha}{\rightarrow} G_{2}^{\prime}, \tau \notin \text { inActs }\left(G_{1}\right), \alpha \notin \text { inActs }\left(G_{1}\right)}{\vdash_{R \cup \text { inActs }\left(G_{1}\right)} G_{1}>G_{2} \stackrel{\leftrightarrow}{\rightarrow} G_{2}^{\prime}} \\
\frac{\vdash_{R} A_{0} \stackrel{\alpha}{\rightarrow} A_{0}^{\prime}, R \cap \text { out } A c t s\left(A_{1}\right)=\emptyset}{\vdash_{R} A_{0}\left|A_{1} \stackrel{\alpha}{\rightarrow} A_{0}^{\prime}\right| A_{1}} \\
\frac{\vdash_{R} A_{1} \stackrel{\alpha}{\rightarrow} A_{1}^{\prime} R \cap \text { outActs }\left(A_{0}\right)=\emptyset}{\vdash_{R} A_{0}\left|A_{1} \stackrel{\alpha}{\rightarrow} A_{0}\right| A_{1}^{\prime}}
\end{gathered}
$$




$$
\begin{gathered}
\frac{\vdash_{R_{0}} A_{0} \stackrel{\alpha}{\rightarrow} A_{0}^{\prime}, \vdash_{R_{1}} A_{1} \stackrel{\stackrel{\alpha}{\rightarrow}}{\rightarrow} A_{1}^{\prime}, R_{1} \cap \text { outActs }\left(A_{0}\right)=\emptyset, R_{0} \cap \operatorname{outActs}\left(A_{1}\right)=\emptyset}{\vdash_{R_{0} \cup R_{1}} A_{0}\left|A_{1} \stackrel{\tau}{\rightarrow} A_{0}^{\prime}\right| A_{1}^{\prime}} \\
\frac{\vdash_{R} A\{f i x(X=A) / X\} \stackrel{\alpha}{\rightarrow} A^{\prime}}{\vdash_{R} \text { fix }(X=A) \stackrel{\alpha}{\rightarrow} A^{\prime}}
\end{gathered}
$$

where $[A / Y]$ means syntactical substitution by $A$ of free occurrences of the variable $Y$. An intuitive explanation of the transition-rules:

- (20) There is no requirement on the refusal set of the environment in order for the first action of a prefix to occur.

- (21) + (22) Correspond to the rules for $C C S$.

- (23) The refusal set has to be changed, because actions in $L \cup \bar{L}$ are not observable, so no environment is able to do a handshake over one of them.

- (24) To the outside world the original refusal set will look like the relabeled one (this works, because $f$ injective implies $f$ bijective).

- (25) The highest prioritized agent may always perform its transitions.

- (26) Since inActs gives every possible action for a guard, the refusal set for $G_{2}$ transitions should exactly be extended with inActs $\left(G_{1}\right)$. Since $\tau$ is not allowed in refusal sets, $\tau \notin \operatorname{inActs}\left(G_{1}\right)$ is a necessary condition.

Furthermore it must be the case that the $G_{2}$ action is not itself in the new refusal set - it does not make sense to have an agent perform an action that is never observable, because the corresponding output action is forbidden by the transition's refusal set. This is consistent with the intuitive description of $\vdash_{R} A \stackrel{\alpha}{\rightarrow} A^{\prime}$ - an environment cannot both refuse $\alpha$ and be prepared to match it.

- $(27)+(28)$ outActs gives exactly every possible output action for an agent, and one of the agents in a parallel composition cannot do a transition if the other one violates the refusal set condition (since the two subagents are part of each other's environments).

- (29) The refusal set for the handshake is the union of the individual refusal sets otherwise one of the handshake transitions would be impossible.

\subsection{Results for the transition system}

Not surprisingly (since an environment cannot both refuse an action and match it) we do indeed have:

Theorem 1 Let $R$ be a refusal set, $\alpha \in A$ ct and $A, A^{\prime} \in A g t:$ If $\vdash_{R} A \stackrel{\alpha}{\rightarrow} A^{\prime}$ then $\alpha \notin R$

The following result is significant because it allows refusal sets to be represented as finite lists: 
Theorem 2 If the agent $A$ respects the given syntax, then any $A$-transition has a finite refusal set.

Furthermore $C C S^{>}$extends $C C S$ (since $C C S$ is a syntactic subclass of $C C S^{>}$):

Theorem 3 Let $A$ be a CCS agent (without any PRISUM that is): $\vdash_{\emptyset} A \stackrel{\alpha}{\rightarrow} A^{\prime}$ iff $A \stackrel{\alpha}{\rightarrow} A^{\prime}$

The relation to Camilleri[90] and Camilleri/Winskel[91] is established by:

Theorem 4 Let $R, R^{\prime}$ be refusal sets, $A, A^{\prime} \in A g t^{J C}, \alpha \in A$ at and $\alpha \notin R^{\prime}$ :

$$
\vdash_{R^{\prime}}^{J C} A \stackrel{\alpha}{\rightarrow} A^{\prime} \quad \text { iff } \quad \exists R \subseteq R^{\prime} . \vdash_{R} A \stackrel{\alpha}{\rightarrow} A^{\prime}
$$

where F $^{J C}$ represents the transition system used in Camilleri[90], and Agt ${ }^{\mathrm{JC}}$ is the class of agents considered in Camilleri[90] (where the syntax is a bit more restrictive than mine).

\section{A new definition of bisimulation and equivalence}

The definition of strong observational equivalence is not quite so obvious as in Milner[89]. Consider the two agents $\mathrm{P}$ and $\mathrm{Q}$ defined as $P=\alpha \cdot A+(\beta . B>\alpha . A)$ and $Q=\alpha . A+\beta . B$. Now we would of course expect $\mathrm{P}$ and $\mathrm{Q}$ to be equivalent, but for $\mathrm{P}$ we get the transition $\vdash_{\{\beta\}} P \stackrel{\alpha}{\rightarrow} A$ which cannot be matched directly by $\mathrm{Q}$, since the only $\alpha$-transition for $\mathrm{Q}$ is $\vdash_{\emptyset} Q \stackrel{\alpha}{\rightarrow} A$ The problem is that several subagents are syntactically equal, and therefore one gets several different refusal sets for the same action and derived agent.

Instead we would expect $P$ and $Q$ to be equivalent, if a transition for one of the agents under refusal set $R$, could be matched by the other agent under refusal set $R^{\prime} \subseteq R$ (remember that refusal sets are considered to be conditions on transitions), since in the above example we do in fact also have $t_{0} P \stackrel{\alpha}{\rightarrow} A$

Note that if every transition can be matched by a smaller or equal refusal set, then regardless of the environment the two agents do in fact have the same behaviour under the intuitive interpretation of $\rightarrow$. For closed agents (no free variables) we are lead to:

Definition 3 The symmetric relation $S \subseteq A g t \times A g t$ is a bisimulation with respect to $\rightarrow$, if $(P, Q) \in S$ implies for all $\alpha \in$ Act, for all refusal sets $R$,

(1) Whenever $\vdash_{R} P \stackrel{\alpha}{\rightarrow} P^{\prime}$ then for some $Q^{\prime}, \exists R^{\prime} . R^{\prime} \subseteq R \wedge \vdash_{R^{\prime}} Q \stackrel{\alpha}{\rightarrow} Q^{\prime} \wedge\left(P^{\prime}, Q^{\prime}\right) \in S$

(2) Whenever $\vdash_{R} Q \stackrel{\alpha}{\rightarrow} Q^{\prime}$ then for some $P^{\prime}, \exists R^{\prime} . R^{\prime} \subseteq R \wedge \vdash_{R^{\prime}} P \stackrel{\alpha}{\rightarrow} P^{\prime} \wedge\left(P^{\prime}, Q^{\prime}\right) \in S$

Definition 4 Define strong observational equivalence by $\sim=\cup\{S \mid S$ is a bisimulation $\}$

Theorem 5 The relation $\sim$ is the largest bisimulation, and $\sim$ is an equivalence relation on closed agents satisfying $P \sim Q$ iff, for all $\alpha \in$ Act, for all refusal sets $R$,

(1) Whenever $\vdash_{R} P \stackrel{\alpha}{\rightarrow} P^{\prime}$ then for some $Q^{\prime}, \exists R^{\prime} . R^{\prime} \subseteq R \wedge \vdash_{R^{\prime}} Q \stackrel{\alpha}{\rightarrow} Q^{\prime} \wedge P^{\prime} \sim Q^{\prime}$

(2) Whenever $\vdash_{R} Q \stackrel{\alpha}{\rightarrow} Q^{\prime}$ then for some $P^{\prime}, \exists R^{\prime} . R^{\prime} \subseteq R \wedge \vdash_{R^{\prime}} \stackrel{\leftrightarrow}{\rightarrow} \stackrel{\leftrightarrow}{\rightarrow} P^{\prime} \wedge P^{\prime} \sim Q^{\prime}$

We use the same approach as in Milner[89] to define strong observational equivalence for agents with free variables: 
Definition 5 Let $A$ and $B$ contain at most variables $\tilde{X} \subseteq V S^{n}$. Then

$$
A \sim B \quad \text { iff } \forall \tilde{P} \subseteq A g t^{n} . A\{\tilde{P} / \tilde{X}\} \sim B\{\tilde{P} / \tilde{X}\}
$$

Note that we could use Definition 5 as the general definition of equivalence, since syntactic substitution for a variable has no effect on closed terms.

We proceed to investigate the properties of $\sim$ :

Theorem $6 \sim$ is a congruence (equivalence is preserved in all algebraic contexts).

For the proof of Theorem 6 the following definitions and theorems are needed (partly analogously with Milner[89]):

Definition 6 The symmetric relation $S \subseteq A g t \times A g t$ is a bisimulation up to $\sim$ with respect to $\rightarrow$, if $(P, Q) \in S$ implies for all $\alpha \in$ Act, for all refusal sets $R$,

(1) Whenever $\vdash_{R} P \stackrel{\alpha}{\rightarrow} P^{\prime}$ then for some $Q^{\prime}, \exists R^{\prime} . R^{\prime} \subseteq R \wedge \vdash_{R^{\prime}} Q \stackrel{\alpha}{\rightarrow} Q^{\prime} \wedge P^{\prime} \sim S \sim Q^{\prime}$

(2) Whenever $\vdash_{R} Q \stackrel{\alpha}{\rightarrow} Q^{\prime}$ then for some $P^{\prime}, \exists R^{\prime} . R^{\prime} \subseteq R \wedge \vdash_{R^{\prime}} \stackrel{\leftrightarrow}{\rightarrow} \stackrel{P^{\prime}}{\wedge} P^{\prime} \sim S \sim Q^{\prime}$

Theorem 7 If $S$ is a bisimulation up to $\sim$ then $\sim S \sim$ is a bisimulation.

Theorem 8 If $S$ is a bisimulation up to $\sim$ then $S \subseteq \sim$.

Theorem 9 If $A \sim B$, then inActs $(A)=\operatorname{inActs}(B) \wedge \operatorname{outActs}(A)=\operatorname{outActs}(B)$.

As a last result (not related to Theorem 6), one might notice

Theorem 10 On Agt ${ }^{J C}$ the equivalence from Camilleri[90] and Camilleri/Winskel[91] coincide with

\section{A modal logic for priorities}

I proceed to define a modal logic for analysing $C C S^{>}$agents. As the labelling of transitions is changed from actions to actions combined with refusal sets, the same is done with the labelling of modalities. The logic then has the following syntax (largely taken from CW):

$$
\begin{gathered}
P::=X|T| F|\sim P| \operatorname{Por} P|\operatorname{Pand} P|<\alpha, R>P|<, R>P|[\alpha, R] P \mid[., R] P \\
|\min (X=P)| \max (X=P)
\end{gathered}
$$

where $T$ and $F$ mean true and false, $\alpha \in A c t$, ' ' means any action, $R$ is a refusal set, $X \in P V$ is a propositional variable, every recursion must be guarded, negative occurrences of recursion variables are forbidden, $\langle\alpha, R\rangle$ and $[\alpha, R]$ denote possibility and necessity respectively, and ' $m$ in' and 'max' denote minimal and maximal fixpoint.

What should the semantic meaning of modal formulae be? Most of the usual semantics can be used, but what about refusal sets? The semantics of refusal sets should have some resemblance to their meaning in the definition of bisimilarity (because intuitively 
the same formulae should be valid for two equivalent agents), so the following definition seems natural:

$$
\begin{aligned}
& A \models<\alpha, R>P \text { iff }(\alpha \notin R) \wedge\left(\exists A^{\prime}, R^{\prime} \subseteq R . \vdash_{R^{\prime}} A \stackrel{\alpha}{\rightarrow} A^{\prime} \wedge A^{\prime} \models P\right) \\
& A \models<., R>P \text { iff } \exists \alpha \in A c t . A \models<\alpha, R>P
\end{aligned}
$$

This agrees with the intuitive idea, that if $A \models<\alpha, R>P \wedge R \subseteq R^{\prime}$, then $A \models<$ $\alpha, R^{\prime}>P$ (if a formula is valid under a condition $R$, then it is also valid under a stronger condition $R^{\prime}$ ). The reason for $\alpha \notin R$ is the implicit relation between $\alpha$ and $R$, that $\alpha$ cannot occur if the environment refuses $\alpha$ (represented by Theorem 1).

Furthermore the reductions $[\alpha, R] P \rightarrow \sim<\alpha, R>\sim P$ and $[., R] P \rightarrow \sim<., R>\sim P$ are needed. The semantics of the rest of the syntax is not changed.

The following characterization theorem then holds:

Theorem 11 The modal logic presented above is characteristic for $C C S^{>}$. That is, for all $P, Q \in$ Agt: $P \sim Q$ iff $\forall$ logic formulae $F .(P \vDash F \Leftrightarrow Q \models F)$

\section{Implementation of priorities in The Concurrency Workbench}

\subsection{Why the Concurrency Workbench?}

$\mathrm{CW}$ is a tool for analysing $C C S$ agents that provides a large number of different analysis methods. It is built in a modular way that better enables changing only the relevant parts of the code, and is state-based so the implemented transition system is relatively easy to find and change.

\subsection{Objectives}

First of all, a necessary objective for such an implementation is that it is conservative - if you do not consider priorities then the new CW should act exactly like the old one. This is achieved by definition and by Theorem 3: If all refusal sets are empty (no priorities are used), then the operational semantics and the logic for $C C S$ and $C C S^{>}$are the same.

Secondly, which parts of CW should in fact be changed? I have chosen for the time being to restrict my attention to the transition system, strong equivalence, logic formulae and model checking.

\subsection{Changes}

The main changes in basic routines and definitions are:

- Module 'BasicAgent': A definition of refusal sets has been added. The PRISUM operator has been added to the datatype representing agents. The function 'transitions', which gives the possible transitions of an agent, has been changed according to the transition system presented above. 
- Module 'AgentIO': The PRISUM operator has been added to the IO-syntax of agents (including the restriction on guards).

- Module 'PolyGraph': It was mainly the function 'mkgraph' (generates stategraphs from agents), that needed change. The list of possible successors to a state under a given action has been changed from a list of states to a list of (refusal set,state). The refusal set from each possible transition is attached to the successor state (not to the action) in order to follow the original structure of CW as closely as possible (thereby minimizing changes and easing the conservativity).

- Module 'PolyGraphOp': The generic equivalence check between agents is done by block partioning (see CW[1] for brief description of algorithm and further references) comparing so-called capabilities (a capability is a list of actions together with a list of blocks reachable through each action). If two states in a block have different capabilities, the block is split in two - two agents are equivalent if they end up in the same block. This function has been changed according to the changes in module 'PolyGraph', and the change in definition of bisimulation presented above. The definition of capabilities has to take refusal sets into account. A list of refusal sets, through which the block is reachable, is attached to each blocknumber (only the smallest possible refusal sets are recorded - two refusal sets belonging to the same block might be included in one another, because a block may contain more than one state).

- Module 'Logics': The definition of the logic for model checking has been changed to suit the modal logic presented above.

- Module 'HMLIO': IO of the modal logic is changed according to the changes in module 'Logics'. The changes are conservative, so that it is possible to use the old logic, and forget all about refusal sets.

- Module 'HMLChecker': The function 'check', that performs the validity check of modal formulae, has been changed according to the semantics for the modal logic presented above, reinterpreting the meaning of the modalities possibility and necessity.

\subsection{Examples of sessions in the new Concurrency Workbench}

The example below shows that PRISUM doesn't distribute over sum. It also illustrates the use of the new modal logic:

Command: strong equivalence

Agent: (a.nil > b.nil) + (c.nil > b.nil)

Agent: (a.nil + c.nil) $>$ b.nil

false

Command: check strong proposition

Agent: (a.nil > b.nil) + (c.nil > b.nil) 
Proposition: $<\mathrm{b},\{\mathrm{a}\}>\mathrm{T}$

true

Command: check strong proposition

Agent: $($ a.nil + c.nil $)>$ b.nil

Proposition: $<\mathrm{b},\{\mathrm{a}\}>\mathrm{T}$

false

Note, that the proposition $<\mathrm{b},\{\mathrm{a}, \mathrm{c}\}>\mathrm{T}$ holds for both the above agents, so larger refusal sets $d o$ in fact make a difference in the logic.

This example shows how to implement a fair (cyclic) critical region (or a scheduler "on need"). Notice, that if only one of the processes wants the critical region, it gets it regardless of whose turn it is.

Command: bind identifier

Identifier: S1

Agent: a.S2 > b.S1

Command: bind identifier

Identifier: S2

Agent: b.S1 > a.S2

Command: check strong proposition

Agent: S1

Proposition: $\max (\mathrm{X} .<\mathrm{a},\{\}>\mathrm{X})$

false

Command: check strong proposition

Agent: S1

Proposition: $\max (\mathrm{X} .<\mathrm{a},\{\mathrm{b}\}>\mathrm{T} \&[\mathrm{a},\{\mathrm{b}\}] \mathrm{X})$

true

\section{Conclusion}

A new $C C S$-constructor $>$ to treat priorities has been proposed (similar to Camilleri[90]). An implementable and hopefully intuitive transition system for the extended $C C S^{>}$has been presented. The definitions of strong bisimilarity and strong equivalence have been changed accordingly. An extended modal logic has been presented. These features have been implemented in an existing analysis tool, in a way that allows the user to work freely with both $C C S$ and $C C S^{>}$. To my knowledge, this is the only existing automated tool for investigating processes with priority. 
In the future, I shall investigate weak bisimulations in connection with priorities, and possibly other equivalence criteria as well.

I plan to extend the work to a generalised analogue of the PRI PAR construct in OCCAM (PRI PAR is fairly restrictive), where it becomes sensible to allow output actions in guards.

\section{Acknowledgements}

Part of this note can be viewed as an extension of the $\mathrm{PhD}$ work done by Juanito Albert Camilleri on the notion of priority in $C C S$. My debt to the work underlying the Concurrency Workbench is clear. I wish to thank Juanito Camilleri and Glynn Winskel for discussions.

\section{Reference list}

Milner[89]: Milner, Robin, Communication and Concurrency, C.A.R. Hoare, Series editor, Prentice Hall, 1989

Winskel[89]: Winskel, Glynn, An Note on Model Checking the Modal $\nu$-calculus, DAIMI PB - 279, Computer Science Department, Aarhus University, 1989

Camilleri[90]: Camilleri, Juanito A., Priority in Process Calculi, Ph.D. Thesis, Computer Laboratory, University of Cambridge, 1991

CW[1]: Cleaveland, R., Parrow, J. and Steffen, B., The Concurrency Workbench: A Semantics Based Tool for the Verification of Concurrent Systems, LFCS Report Series, ECS-LFCS-89-83, Department of Computer Science, University of Edinburgh, 1989

CW[2]: Cleaveland, R., Parrow, J. and Steffen, B., The Concurrency Workbench: Operating Instructions, LFCS-TN-10, 1988

CW[3]: Morley, Matthew J., Tactics for State Space Reduction on the Concurrency Workbench, LFCS Report Series, ECS-LFCS-90-109, Department of Computer Science, University of Edinburgh, 1990

OCC[84]: inmos. OCCAM Programming Manual, International Series in Computer Science, Prentice Hall, 1984

Camilleri/Winskel[91]: Camilleri, Juanito A., Winskel, Glynn, CCS with priority choice, to appear in proceedings of LICS 91 